\title{
Article \\ Stieltjes Differential Inclusions with Periodic Boundary Conditions without Upper Semicontinuity
}

\author{
Valeria Marraffa $^{1}$ (D) and Bianca Satco ${ }^{2, *(D)}$ \\ 1 Dipartimento di Matematica e Informatica, Università degli Studi di Palermo, Via Archirafi 34, \\ 90123 Palermo, Italy; valeria.marraffa@unipa.it \\ 2 Faculty of Electrical Engineering and Computer Science, Stefan cel Mare University of Suceava, \\ Universitatii 13, 720225 Suceava, Romania \\ * Correspondence: bisatco@usm.ro
}

check for updates

Citation: Marraffa, V.; Satco, B. Stieltjes Differential Inclusions with Periodic Boundary Conditions without Upper Semicontinuity. Mathematics 2022, 10, 55. https:// doi.org/10.3390/math10010055

Academic Editor: Snezhana Hristova

Received: 1 December 2021 Accepted: 22 December 2021 Published: 24 December 2021

Publisher's Note: MDPI stays neutral with regard to jurisdictional claims in published maps and institutional affiliations.

Copyright: (C) 2021 by the authors. Licensee MDPI, Basel, Switzerland. This article is an open access article distributed under the terms and conditions of the Creative Commons Attribution (CC BY) license (https:// creativecommons.org/licenses/by/ $4.0 /)$.

\begin{abstract}
We are studying first order differential inclusions with periodic boundary conditions where the Stieltjes derivative with respect to a left-continuous non-decreasing function replaces the classical derivative. The involved set-valued mapping is not assumed to have compact and convex values, nor to be upper semicontinuous concerning the second argument everywhere, as in other related works. A condition involving the contingent derivative relative to the non-decreasing function (recently introduced and applied to initial value problems by R.L. Pouso, I.M. Marquez Albes, and J. Rodriguez-Lopez) is imposed on the set where the upper semicontinuity and the assumption to have compact convex values fail. Based on previously obtained results for periodic problems in the single-valued cases, the existence of solutions is proven. It is also pointed out that the solution set is compact in the uniform convergence topology. In particular, the existence results are obtained for periodic impulsive differential inclusions (with multivalued impulsive maps and finite or possibly countable impulsive moments) without upper semicontinuity assumptions on the right-hand side, and also the existence of solutions is derived for dynamic inclusions on time scales with periodic boundary conditions.
\end{abstract}

Keywords: differential inclusion; periodic boundary value condition; Stieltjes derivative; impulse; dynamic equation on time scales

MSC: 34A06; 28B20; 34B37; 34N05

\section{Introduction}

The theory of differential equations involving the Stieltjes derivative [1] instead of the usual derivative has recently developed significantly (we refer to [2-4] and the references therein). The reason is the fact that it offers another point of view on the theory of measure differential problems [1,5-8] and, in particular cases, it yields new results for ordinary differential equations, for difference equations, or for impulsive differential equations [1-3]. At the same time, it allows one to assert new results for the (also recent) theories of dynamic equations on time scales and generalized differential equations $[7,9,10]$. This is the main reason for the use of Stieltjes derivatives.

On the other hand, it is clear that passing from single-valued problems to the setvalued setting [11-15], thus studying Stieltjes differential inclusions, widens the field of possible applications of theoretical results.

Aware of the significance of periodic boundary conditions when studying real life processes, the present work focuses on the analysis of a first-order differential inclusion with periodic boundary value conditions

$$
\left\{\begin{array}{l}
u_{g}^{\prime}(t)+p(t) u(t) \in F(t, u(t)), \mu_{g}-\text { a.e. } t \in[0, T] \\
u(0)=u(T)
\end{array}\right.
$$


involving the Stieltjes derivative with respect to a left-continuous non-decreasing function $g:[0, T] \rightarrow \mathbb{R}$ and a real function $p$, Lebesgue-Stieltjes integrable with respect to $g$.

This problem was investigated in the single-valued framework in [16] and then generalized to the multivalued setting in [17] under the assumptions that $F:[0, T] \times \mathbb{R}^{d} \rightarrow \mathcal{P}\left(\mathbb{R}^{d}\right)$ has convex, compact values and it is a Carathéodory multifunction.

Applying now an idea presented (and used in the study of initial value problems) in [4], we prove that the existence results in [17] can be obtained in the less restrictive case where $F$ is convex, compact-valued, and upper semi-continuous with respect to its second variable, except for a set (which can be dense in $\mathbb{R}^{d}$, e.g., Example 3.11 in [4]), where a condition involving the notion of contingent $g$-derivative must be imposed. We also obtain the compactness (in the topology of uniform convergence) of the solution set.

The technical tool used in the proof is a successive approximation, combined with weak compactness criteria in the space of Lebesgue-integrable functions.

By taking specific nondecreasing functions $g$, we then derive the existence of solutions for impulsive differential inclusions with periodic boundary assumptions, in both cases, the number of abrupt perturbations being finite or countable (i.e., allowing Zeno behavior, see [18]) and present an example to illustrate the wide applicability of the developed study. The result provided includes already published results, see for instance [14,19-21] or [13] (alternative studies for this kind of problems concern equations with variable impulsive time, e.g., [22], fractional periodic first-order impulsive inclusions, e.g., [23] or problems with constraints, such as $[24,25])$. The generalization is double: besides imposing convex, compact values and upper semicontinuity everywhere, in all these works the impulsive maps are single-valued, while we allow set-valued impulsive mappings.

Finally, given the equivalence (under a rather convenient hypothesis) of the theory of Stieltijes differential equations with that of measure differential equations $[1,3]$ and consequently (cf. [7], see also [9]) with the theory of dynamic equations on time scale domains, we derive an existence result for dynamic inclusions on bounded time scales with periodic boundary conditions. We thus generalize several results in the literature, such as the multivalued results in $[10,26,27]$ (or the single-valued results in $[28,29]$ ) where the multifunction on the right-hand side was supposed to be convex, compact-valued, and upper semicontinuous concerning the second argument.

\section{Preliminaries}

A function $u:[0, T] \rightarrow \mathbb{R}^{d}$ having limits $u(t+)$ and $u(s-)$ at every points $t \in[0, T)$, $s \in(0, T]$ is regulated. A collection $\mathcal{A}$ of regulated functions is said to be equiregulated if for every $\varepsilon>0$ and every $t_{0} \in(0, T]$ there exists $\delta_{\varepsilon, t_{0}}>0$ such that for all $u \in \mathcal{A}$ : $\left\|u(t)-u\left(t_{0}-\right)\right\|<\varepsilon$ whenever $t_{0}-\delta<t<t_{0}$ (and similarly at the right).

In [30] (Theorem 5.1) it was proved the following Ascoli-type theorem.

Theorem 1. If an equiregulated sequence of maps converges pointwise, then the convergence is uniform.

Let $D_{g}$ be the set of the discontinuity points of the nondecreasing left-continuous function $g$ (which is at most countable). Then the measurability w.r.t. the $\sigma$-algebra defined by $g$ will be called $g$-measurability, $\mu_{g}$ stands for the Stieltjes measure generated by $g$ and the Lebesgue-Stieltjes (shortly, LS-) integrability w.r.t. $g$ means the abstract Lebesgue integrability w.r.t. the Stieltjes measure $\mu_{g}$. Let $L_{g}^{1}([0, T])$ be the space of real functions LS-integrable w.r.t. $g$ on $[0, T]$. It is well known that if $f \in L_{g}^{1}\left([0, T], \mathbb{R}^{d}\right)$, the primitive $\int_{0}^{\cdot} f(s) d g(s)=\int_{[0, \cdot)} f(s) d g(s)$ is a function of bounded variation, therefore it is regulated.

Let us see an auxiliary result. 
Lemma 1. Let $\mathcal{A}$ be a collection of $\mathbb{R}^{d}$-valued functions on $[0, T]$, LS-integrable w.r.t. g. If there exists $\phi \in L_{g}^{1}([0, T])$ satisfying

$$
\|u(t)\| \leq \phi(t), \mu_{g}-\text { a.e., for all } u \in \mathcal{A},
$$

then the set of primitives $\left\{\int_{0}^{\cdot} u(s) d g(s): u \in \mathcal{A}\right\}$ is equiregulated.

Proof. Let $\varepsilon>0$ and $t_{0} \in[0, T)$. Then there is $\delta_{\varepsilon, t_{0}}>0$ such that

$$
\left\|\int_{0}^{t} \phi(s) d g(s)-\int_{0}^{t_{0}+} \phi(s) d g(s)\right\|<\varepsilon
$$

whenever $t_{0}<t<t_{0}+\delta_{\varepsilon, t_{0}}$.

It immediately follows that, for every $u \in \mathcal{A}$,

$$
\left\|\int_{0}^{t} u(s) d g(s)-\int_{0}^{t_{0}+} u(s) d g(s)\right\| \leq \int_{t}^{t_{0}+}\|u(s)\| d g(s) \leq \int_{t}^{t_{0}+} \phi(s) d g(s)<\varepsilon
$$

for all $t_{0}<t<t_{0}+\delta_{\varepsilon, t_{0}}$.

As the inequality for the left limit can be proved in the same way, the equiregulatedness is verified.

In our study, the usual derivative will be replaced by a notion of Stieltjes derivative ([1], see also [31]) which has already found significant applications in real life problems (e.g., $[2,32,33])$. The Stieltjes or $g$-derivative of a function $u:[0, T] \rightarrow \mathbb{R}^{d}$ with respect to $g$ at a point $t \in[0, T]$ is (in the case of existence of limits)

$$
\begin{aligned}
& u_{g}^{\prime}(t)=\lim _{t^{\prime} \rightarrow t} \frac{u\left(t^{\prime}\right)-u(t)}{g\left(t^{\prime}\right)-g(t)} \quad \text { if } g \text { is continuous at } t, \\
& u_{g}^{\prime}(t)=\lim _{t^{\prime} \rightarrow t+} \frac{u\left(t^{\prime}\right)-u(t)}{g\left(t^{\prime}\right)-g(t)} \quad \text { if } t \in D_{g} .
\end{aligned}
$$

Note that if $t \in D_{g}$, it suffices that $u$ have right limit $u(t+)$ in order to have

$$
u_{g}^{\prime}(t)=\frac{u(t+)-u(t)}{g(t+)-g(t)} .
$$

The particular cases of the theory of Stieltjes differential equations are coming from taking particular non-decreasing functions $g$. More precisely: when $g(t)=t$ we get ordinary differential equations, in the case where $g$ is a sum of step functions we retrieve difference equations, while for the situation where $g$ is a sum of the identical function with step functions we arrive at impulsive problems. This is the advantage of using Stieltjes derivatives: we are studying various problems under one roof.

The following result concerning the $g$-derivative of a limit of a sequence of $g$-absolutely continuous functions will be useful in the main proof. A function $u:[0, T] \rightarrow \mathbb{R}^{d}$ is $g$ absolutely continuous (see [34] or [1]) if for every $\varepsilon>0$ there is $\delta_{\varepsilon}>0$ such that

$$
\sum_{j=1}^{m}\left\|u\left(t_{j}^{\prime \prime}\right)-u\left(t_{j}^{\prime}\right)\right\|<\varepsilon
$$

for any set $\left\{\left(t_{j}^{\prime}, t_{j}^{\prime \prime}\right)\right\}_{i=1}^{m}$ of non-overlapping subintervals of $[0, T]$ with $\sum_{j=1}^{m}\left(g\left(t_{j}^{\prime \prime}\right)-g\left(t_{j}^{\prime}\right)\right)<\delta_{\varepsilon}$.

Lemma 2 ([4] (Lemma 3.8)). Let $\left(u_{k}\right)_{k}$ be a sequence of $g$-absolutely continuous $\mathbb{R}^{d}$-valued functions on $[0, T]$, pointwisely convergent to some $u:[0, T] \rightarrow \mathbb{R}^{d}$. If there exists $\phi \in L_{g}^{1}([0, T])$ such that for all $k \in \mathbb{N}$,

$$
\left\|\left(u_{k}\right)_{g}^{\prime}(t)\right\| \leq \phi(t), \mu_{g}-\text { a.e., }
$$


then $u$ is also g-absolutely continuous and

$$
u_{g}^{\prime}(t) \in \bigcap_{i \in \mathbb{N}} \overline{c o} \bigcup_{k=i}^{\infty}\left\{\left(u_{k}\right)_{g}^{\prime}(t)\right\}, \mu_{g}-\text { a.e. }
$$

We rely on an existence result provided in [17] for the single-valued linear periodic problem

$$
\left\{\begin{array}{l}
u_{g}^{\prime}(t)+p(t) u(t)=f(t), \quad \mu_{g} \text {-a.e. in }[0, T], \\
u(0)=u(T),
\end{array}\right.
$$

under the nonresonance assumption:

$$
1-p(t) \mu_{g}(\{t\}) \neq 0, \quad \text { for every } t \in[0, T] \text {. }
$$

An important matter is to realize that the sign of $1-p(t) \mu_{g}(\{t\})$ has to be considered. As in [2], the set

$$
D_{g}^{-}=\left\{t \in D_{g}: 1-p(t) \mu_{g}(\{t\})<0\right\}
$$

is finite and, if we denote its elements by $t_{1}<\ldots<t_{k}$ (and, for the sake of convenience, take $t_{0}=0$ and $t_{k+1}=T$ ), we define

$$
\alpha(t)=\left\{\begin{array}{l}
1, \text { if } t \in\left[0, t_{1}\right] \\
(-1)^{i}, \text { if } t \in\left(t_{i}, t_{i+1}\right], i=1, \ldots, k
\end{array}\right.
$$

and

$$
\tilde{p}(t)=\left\{\begin{array}{l}
p(t), \text { if } g \text { continuous at } t \\
\frac{-\log \left|1-p(t) \mu_{g}(\{t\})\right|}{\mu_{g}(\{t\})}, \text { if } t \in D_{g} .
\end{array}\right.
$$

Theorem 2 ([17] (Theorem 2, Remarks 2 and 3)). Let $f \in L_{g}^{1}\left([0, T], \mathbb{R}^{d}\right)$. Then the problem (2) has a unique solution,

$$
u(t)=\frac{1}{\alpha(t)} \int_{0}^{T} \frac{\alpha(s)}{1-p(s) \mu_{g}(\{s\})} \tilde{g}(t, s) f(s) d g(s),
$$

where

$$
\tilde{g}(t, s)=\frac{1}{\alpha(T) e^{\int_{0}^{T} \tilde{p}(r) d g(r)}-1}\left\{\begin{array}{l}
\alpha(T) e^{\int_{0}^{T} \tilde{p}(r) d g(r)-\int_{s}^{t} \tilde{p}(r) d g(r)}, \text { if } 0 \leq s \leq t \leq T \\
e^{-\int_{s}^{t} \tilde{p}(r) d g(r)}, \text { if } 0 \leq t<s \leq T .
\end{array}\right.
$$

Let us remind [16] of the fact that the mapping $\left(s^{\prime}, s^{\prime \prime}\right) \in[0, T]^{2} \mapsto e^{\int_{s^{\prime}}^{s^{\prime \prime}}} \tilde{p}(s) d g(s)$ is bounded, say by

$$
M=\sup _{\left(s^{\prime}, s^{\prime \prime}\right) \in[0, T]^{2}} e^{\int_{s^{\prime}}^{s^{\prime \prime}} \tilde{p}(s) d g(s)} .
$$

Moreover there are two positive constants $\delta, \bar{\delta}$ such that

$$
\delta<\left|1-p(t) \mu_{g}(\{t\})\right|<\bar{\delta}, \forall t \in D_{g},
$$

whence

$$
\min \left(1, \frac{1}{\bar{\delta}}\right) \leq \frac{1}{\left|1-p(t) \mu_{g}(\{t\})\right|} \leq \max \left(1, \frac{1}{\delta}\right) \quad \text { for every } t \in[0, T]
$$

As we place ourselves in the multivalued framework, some basic notions of set-valued analysis are needed. 
A compact, convex-valued mapping $\Gamma: I_{\Gamma} \subset \mathbb{R} \rightarrow \mathcal{P}\left(\mathbb{R}^{d}\right)$ is upper semicontinuous at $u \in I_{\Gamma}$ if, for every $\varepsilon>0$, there exists $\delta_{\varepsilon, u}>0$ satisfying the inclusion $\Gamma(v) \subset \Gamma(u)+B_{\varepsilon}(0)$, for any $v \in B_{\delta}(u)$.

Let

$$
\text { Graph } \Gamma=\left\{(t, x) ; t \in I_{\Gamma}, x \in \Gamma(t)\right\} .
$$

Remind the reader of a concept which generalizes the Bouligand's contingent cone to a given set ([11], page 177) to multifunctions: the contingent derivative [35] of $\Gamma$ at $\left(t_{0}, x_{0}\right) \in G r a p h \Gamma$. It is the set-valued map $D \Gamma\left(t_{0}, x_{0}\right): \mathbb{R} \rightarrow \mathcal{P}\left(\mathbb{R}^{d}\right)$ defined as follows: $D \Gamma\left(t_{0}, x_{0}\right)(u)$ contains all $v \in \mathbb{R}^{d}$ for which there exist a sequence $\left(t_{n}, x_{n}\right)_{n} \subset G r a p h \Gamma$ convergent to $\left(t_{0}, x_{0}\right)$ and a sequence $\left(h_{n}\right)_{n} \in \mathbb{R}_{+}$with

$$
\lim _{n \rightarrow \infty} h_{n} \cdot\left(t_{n}-t_{0}\right)=u \text { and } \lim _{n \rightarrow \infty} h_{n} \cdot\left(x_{n}-x_{0}\right)=v .
$$

A single-valued function $\gamma: I_{\Gamma} \rightarrow \mathbb{R}^{d}$ satisfying $\gamma(t) \in \Gamma(t)$ almost everywhere is called a selection of $\Gamma$. Finally, we denote, for a set $A \subset \mathbb{R}^{d}$, by

$$
|A|=\sup \{\|u\| ; u \in A\} \text {. }
$$

\section{Main Result}

Consider, as announced, the set-valued Stieltjes differential problem with periodic boundary condition

$$
\left\{\begin{array}{l}
u_{g}^{\prime}(t)+p(t) u(t) \in F(t, u(t)), \mu_{g} \text {-a.e. } t \in[0, T] \\
u(0)=u(T) .
\end{array}\right.
$$

The concept of solution we are searching for is given in [17] (Definition 4): $u:[0, T] \rightarrow$ $\mathbb{R}^{d}$ is a solution of problem (1) if it is $g$-absolutely continuous and satisfies

$$
u_{g}^{\prime}(t)+p(t) u(t)=f(t)
$$

with $f(t) \in F(t, u(t)), \mu_{g}-$ a.e. in $[0, T]$, together with the equality $u(0)=u(T)$.

In [17] (Theorem 4) the existence of solutions of (1) is proved under the hypotheses that $F$ is convex, compact-valued and upper semicontinuous with respect to its second argument.

We now prove that these assumptions can be relaxed, by using the notion of contingent $g$-derivative, recently introduced in [4].

Definition 1. Let $\Gamma: I_{\Gamma} \subset \mathbb{R} \rightarrow \mathcal{P}\left(\mathbb{R}^{d}\right)$. The contingent g-derivative of $\Gamma$ at $\left(t_{0}, x_{0}\right) \in G$ raph $\Gamma$ is the set $D_{g} \Gamma\left(t_{0}, x_{0}\right)$ containing all $y \in \mathbb{R}^{d}$ for which there exists a sequence $\left(l_{k}\right)_{k} \in \mathbb{R}_{+}$convergent to 0 , such that $\left(t_{0}+l_{k}, x_{k}\right)_{k} \subset$ Graph $\Gamma$ and

$$
\lim _{k \rightarrow \infty} \frac{x_{k}-x_{0}}{g\left(t_{0}+l_{k}\right)-g\left(t_{0}\right)}=y
$$

In the single-valued case, one falls on the concept of $g$-derivative (see [4]), while in the case where $g(t)=t$ one gets the usual contingent derivative for $u=1, D \Gamma\left(t_{0}, x_{0}\right)(1)$.

Indeed, if $v \in D \Gamma\left(t_{0}, x_{0}\right)(1)$, one can find a sequence $\left(t_{n}, x_{n}\right)_{n} \subset G r a p h \Gamma$ convergent to $\left(t_{0}, x_{0}\right)$ and a sequence $\left(h_{n}\right)_{n} \in \mathbb{R}_{+}$with

$$
\lim _{n \rightarrow \infty} h_{n} \cdot\left(t_{n}-t_{0}\right)=1 \text { and } \lim _{n \rightarrow \infty} h_{n} \cdot\left(x_{n}-x_{0}\right)=v \text {. }
$$

Then

$$
v=\lim _{n \rightarrow \infty} h_{n} \cdot\left(x_{n}-x_{0}\right)=\lim _{n \rightarrow \infty} \frac{x_{n}-x_{0}}{t_{n}-t_{0}}\left(t_{n}-t_{0}\right) h_{n}=\lim _{n \rightarrow \infty} \frac{x_{n}-x_{0}}{t_{n}-t_{0}} \in D_{g(t)=t} \Gamma\left(t_{0}, x_{0}\right) .
$$


Conversely, if $v \in D_{g(t)=t} \Gamma\left(t_{0}, x_{0}\right)$, there exists a sequence $\left(l_{k}\right)_{k} \in \mathbb{R}_{+}$convergent to 0 such that $\left(t_{0}+l_{k}, x_{k}\right)_{k} \subset$ Graph $\Gamma$ and

$$
\lim _{k \rightarrow \infty} \frac{x_{k}-x_{0}}{t_{0}+l_{k}-t_{0}}=v \text {. }
$$

So there exist $\left(t_{k}=t_{0}+l_{k}, x_{k}\right)_{k} \subset G r a p h \Gamma$ convergent to $\left(t_{0}, x_{0}\right)$ and $h_{k}=\frac{1}{l_{k}}>0$ such that

$$
1=\lim _{k \rightarrow \infty} h_{k}\left(t_{k}-t_{0}\right) \quad \text { and } \quad v=\lim _{k \rightarrow \infty} h_{k}\left(x_{k}-x_{0}\right),
$$

whence $v \in D \Gamma\left(t_{0}, x_{0}\right)(1)$.

Theorem 3. Let $F:[0, T] \times \mathbb{R}^{d} \rightarrow \mathcal{P}\left(\mathbb{R}^{d}\right)$ satisfy the following hypotheses.

Hypothesis 1. for every regulated function $u:[0, T] \rightarrow \mathbb{R}^{d}, F(\cdot, u(\cdot))$ has g-measurable selections;

Hypothesis 2. (i) for every $t \in[0, T] \cap D_{g}, F(t, \cdot)$ is convex and compact-valued and it is upper semicontinuous;

(ii) for $\mu_{g}$-a.e. $t \in[0, T] \backslash D_{g}, F(t, \cdot)$ is convex compact-valued and upper semicontinuous on $\mathbb{R}^{d} \backslash K(t)$, where the set $K(t)$ is empty or there exists a countable family of maps $K_{j}: \mathcal{I}_{j} \subset$ $[0, T] \backslash D_{g} \rightarrow \mathcal{P}\left(\mathbb{R}^{d}\right), j \in \mathbb{N}$, such that

$$
K(t)=\bigcup_{j: t \in \mathcal{I}_{j}} K_{j}(t)
$$

and whenever $u \in K_{j}(t)$,

$$
\left(\bigcap_{\varepsilon>0} \overline{c o} F\left(t, B_{\varepsilon}(u)\right)-p(t) u\right) \cap D_{g} K_{j}(t, u) \subset F(t, u)-p(t) u ;
$$

Hypothesis 3. there is a function $\phi$, LS-integrable w.r.t. g, such that

$$
|F(t, u)| \leq \phi(t)
$$

for $\mu_{g}$-almost all $t \in[0, T]$ and all $u \in \mathbb{R}^{d}$.

Then the periodic Stieltjes differential inclusion (1) admits solutions. Besides, the solution set is compact in the space of g-absolutely continuous functions with respect to the uniform convergence topology.

Proof. Let $u_{0} \in \mathbb{R}^{d}$ and choose a $g$-measurable selection $f_{0}$ of $F\left(\cdot, u_{0}\right)$. By Hypothesis 3 , $f_{0} \in L_{g}^{1}\left([0, T], \mathbb{R}^{d}\right)$ and so, Theorem 2 yields the existence of a unique solution $u_{1}$ of the periodic Stieltjes differential problem

$$
\left\{\begin{array}{l}
u_{g}^{\prime}(t)+p(t) u(t)=f_{0}(t), \quad \mu_{g}-\text { a.e. in }[0, T] \\
u(0)=u(T)
\end{array}\right.
$$

namely

$$
u_{1}(t)=\frac{1}{\alpha(t)} \int_{0}^{T} \frac{\alpha(s)}{1-p(s) \mu_{g}(\{s\})} \tilde{g}(t, s) f_{0}(s) d g(s), t \in[0, T] .
$$


Next, let $f_{1}$ be a $g$-measurable (therefore, LS-integrable w.r.t. $g$ ) selection of $F\left(\cdot, u_{1}(\cdot)\right)$. By Theorem 2, there exists a unique solution $u_{2}$ of problem

$$
\left\{\begin{array}{l}
u_{g}^{\prime}(t)+p(t) u(t)=f_{1}(t), \quad \mu_{g}-\text { a.e. in }[0, T] \\
u(0)=u(T)
\end{array}\right.
$$

given by

$$
u_{2}(t)=\frac{1}{\alpha(t)} \int_{0}^{T} \frac{\alpha(s)}{1-p(s) \mu_{g}(\{s\})} \tilde{g}(t, s) f_{1}(s) d g(s), t \in[0, T]
$$

and we continue the process: for each $n \geq 2$, choose a selection $f_{n} \in L_{g}^{1}\left([0, T], \mathbb{R}^{d}\right)$ of $F\left(\cdot, u_{n}(\cdot)\right)$. There exists a unique solution $u_{n+1}$ of problem

$$
\left\{\begin{array}{l}
u_{g}^{\prime}(t)+p(t) u(t)=f_{n}(t), \quad \mu_{g}-\text { a.e. in }[0, T] \\
u(0)=u(T)
\end{array}\right.
$$

given by

$$
u_{n+1}=\frac{1}{\alpha(t)} \int_{0}^{T} \frac{\alpha(s)}{1-p(s) \mu_{g}(\{s\})} \tilde{g}(t, s) f_{n}(s) d g(s), t \in[0, T] .
$$

It can be written as

$$
\begin{gathered}
u_{n+1}=\frac{1}{\alpha(T) e^{\int_{0}^{T} \tilde{p}(r) d g(r)}-1} \\
{\left[\frac{\alpha(T)}{\alpha(t)} e^{\int_{0}^{T} \tilde{p}(r) d g(r)} e^{-\int_{0}^{t} \tilde{p}(r) d g(r)} \int_{0}^{t} \frac{\alpha(s) e \int_{0}^{s} \tilde{p}(r) d g(r)}{1-p(s) \mu_{g}(\{s\})} \cdot f_{n}(s) d g(s)\right.} \\
\left.+\frac{1}{\alpha(t)} e^{-\int_{0}^{t} \tilde{p}(r) d g(r)} \int_{t}^{T} \frac{\alpha(s) e^{\int_{0}^{s} \tilde{p}(r) d g(r)}}{1-p(s) \mu_{g}(\{s\})} \cdot f_{n}(s) d g(s)\right]
\end{gathered}
$$

The sequence $\left(f_{n}\right)_{n}$ is relatively compact in the weak topology of $L_{g}^{1}\left([0, T], \mathbb{R}^{d}\right)$ by (Hypothesis 3), therefore, one can find a subsequence (not relabelled) convergent in this topology to some $f \in L_{g}^{1}\left([0, T], \mathbb{R}^{d}\right)$. As for all $t \in[0, T]$

$$
\left|\frac{\alpha(t) e^{\int_{0}^{t} \tilde{p}(r) d g(r)}}{1-p(t) \mu_{g}(\{t\})}\right| \leq \max \left(1, \frac{1}{\delta}\right) \cdot M,
$$

we derive that, for each $t \in[0, T]$,

$$
\begin{aligned}
& \int_{0}^{t} \frac{\alpha(s) e^{\int_{0}^{s} \tilde{p}(r) d g(r)}}{1-p(s) \mu_{g}(\{s\})} \cdot f_{n}(s) d g(s) \rightarrow \int_{0}^{t} \frac{\alpha(s) e^{\int_{0}^{s} \tilde{p}(r) d g(r)}}{1-p(s) \mu_{g}(\{s\})} \cdot f(s) d g(s), \\
& \int_{t}^{T} \frac{\alpha(s) e_{0}^{\int_{0}^{s} \tilde{p}(r) d g(r)}}{1-p(s) \mu_{g}(\{s\})} \cdot f_{n}(s) d g(s) \rightarrow \int_{t}^{T} \frac{\alpha(s) e_{0}^{\int_{0}^{s}} \tilde{p}(r) d g(r)}{1-p(s) \mu_{g}(\{s\})} \cdot f(s) d g(s) .
\end{aligned}
$$

So, $\left(u_{n}\right)_{n}$ tends pointwise to $u:[0, T] \rightarrow \mathbb{R}^{d}$ defined by

$$
\begin{gathered}
u(t)=\frac{1}{\alpha(T) e^{\int_{0}^{T} \tilde{p}(r) d g(r)}-1} \\
{\left[\frac{\alpha(T)}{\alpha(t)} e^{\int_{0}^{T} \tilde{p}(r) d g(r)} e^{-\int_{0}^{t} \tilde{p}(r) d g(r)} \int_{0}^{t} \frac{\alpha(s) e^{\int_{0}^{s} \tilde{p}(r) d g(r)}}{1-p(s) \mu_{g}(\{s\})} \cdot f(s) d g(s)\right.} \\
\left.+\frac{1}{\alpha(t)} e^{-\int_{0}^{t} \tilde{p}(r) d g(r)} \int_{t}^{T} \frac{\alpha(s) e_{0}^{s} \tilde{p}(r) d g(r)}{1-p(s) \mu_{g}(\{s\})} \cdot f(s) d g(s)\right]
\end{gathered}
$$


Again by Theorem 2, $u$ is the unique solution of

$$
\left\{\begin{array}{l}
u_{g}^{\prime}(t)+p(t) u(t)=f(t), \quad \mu_{g}-\text { a.e. in }[0, T], \\
u(0)=u(T) .
\end{array}\right.
$$

Let us check that it is a solution of our differential inclusion (1). By basic properties of Lebesgue-Stieltjes integrals, it is $g$-absolutely continuous.

Since the sequence $\left(f_{n}\right)_{n} \subset L_{g}^{1}\left([0, T], \mathbb{R}^{d}\right)$ is relatively weakly compact, one can find a sequence of convex combinations of $\left\{f_{m}: m \geq n\right\} \mu_{g}$-a.e. convergent to $f$, so let us consider a $\mu_{g}$-null set $E \subset[0, T]$, such that, for every $t \in[0, T] \backslash E, u_{g}^{\prime}(t)+b(t) u(t)=f(t)$ and the specified sequence of convex combinations of $\left\{f_{m}: m \geq n\right\}$ is convergent, at the point $t$, to $f(t)$.

Define for each $j \in \mathbb{N}$ the set

$$
\begin{aligned}
\tilde{K}_{j}= & \left\{t \in[0, T] \backslash\left(E \cup D_{g}\right): u(t) \in K_{j}(t),\right. \\
& \left.u(s) \notin K_{j}(s) \text { for all } s \in(t, t+\delta(t)) \text { with } \delta(t)>0\right\} .
\end{aligned}
$$

As in [4], $\mu_{g}\left(\tilde{K}_{j}\right)=0$. Then the set

$$
\tilde{E}=E \cup \bigcup_{j=1}^{\infty} \tilde{K}_{j}
$$

is of $\mu_{g}$-null measure and suppose Hypothesis 2.(ii) is verified except for $t \in \tilde{E} \cup D_{g}$.

Take now $t \in[0, T] \backslash \tilde{E}$ and consider, in order to prove that

$$
u_{g}^{\prime}(t)+p(t) u(t) \in F(t, u(t))
$$

the following possible situations.

Case I. $t \in D_{g}$ or $\left(t \in[0, T] \backslash\left(\tilde{E} \cup D_{g}\right)\right.$ and $\left.u(t) \notin K(t)\right)$. Then $F(t, u(t))$ is convex and compact and $F(t, \cdot)$ is upper semicontinuous at $u(t)$. It means that for every $\varepsilon>0$ there is $\delta_{\varepsilon, t}>0$ such that

$$
F(t, y) \subset F(t, u(t))+B_{\varepsilon}(0), \text { for all } y \in B_{\delta}(u(t)) .
$$

Consequently, for every $\varepsilon>0$ one can find $n_{\varepsilon, t} \in \mathbb{N}$ such that

$$
F\left(t, u_{n}(t)\right) \subset F(t, u(t))+B_{\varepsilon}(0), \text { for all } n \geq n_{\varepsilon, t} .
$$

Since $f(t)$ is a limit of convex combinations of $\left\{f_{m}(t): m \geq n\right\}$ it follows that, in this case, $u_{g}^{\prime}(t)+p(t) u(t)=f(t) \in F(t, u(t))$.

Case II. $t \in[0, T] \backslash\left(\tilde{E} \cup D_{g}\right)$ and $u(t) \in K(t)$. There exists some $j \in \mathbb{N}$ such that $u(t) \in K_{j}$. Obviously, $t \notin \tilde{K}_{j}$ and so, there exists a sequence $\left(l_{k}\right)_{k} \subset \mathbb{R}_{+}$convergent to 0 such that $u\left(t+l_{k}\right) \in K_{j}\left(t+l_{k}\right)$ for all $k$ and, as $u$ is $g$-differentiable at $t$, by Definition 1 ,

$$
u_{g}^{\prime}(t)=\lim _{k \rightarrow \infty} \frac{u\left(t+l_{k}\right)-u(t)}{g\left(t+l_{k}\right)-g(t)} \in D_{g} K_{j}(t, u(t)) .
$$

At the same time, Lemma 2 implies that

$$
u_{g}^{\prime}(t) \in \bigcap_{i \in \mathbb{N}} \overline{c o} \bigcup_{k=i}^{\infty}\left\{\left(u_{k}\right)_{g}^{\prime}(t)\right\} \mu_{g}-\text { a.e. }
$$

For every $\varepsilon>0$, there exists $i_{\varepsilon} \in \mathbb{N}$ (depending on $t$ ) such that

$$
\left\|u_{k}(t)-u(t)\right\| \leq \varepsilon, \text { for all } k \geq i_{\varepsilon}
$$


and, as

$$
u_{g}^{\prime}(t) \in \overline{c o} \bigcup_{k=i_{\varepsilon}}^{\infty}\left\{\left(u_{k}\right)_{g}^{\prime}(t)\right\}
$$

it is the limit, as $m \rightarrow \infty$, of a sequence $\left(y_{m}(t)\right)_{m}$ such that for each $m \in \mathbb{N}, y_{m}(t) \in$ co $\bigcup_{k=i_{\varepsilon}}^{\infty}\left\{\left(u_{k}\right)_{g}^{\prime}(t)\right\}$. For each $m$ one can thus find $\tilde{l}_{m} \in \mathbb{N}$ and $\lambda_{j}^{m}>0, j=1, \ldots, \tilde{l}_{m}$ with $\sum_{j=1}^{\tilde{l}_{m}} \lambda_{j}^{m}=1$ and also $\alpha_{j}^{m} \in \mathbb{N}, j=1, \ldots, \tilde{l}_{m}$ such that

$$
y_{m}(t)=\sum_{j=1}^{\tilde{l}_{m}} \lambda_{j}^{m}\left(u_{i_{\varepsilon}+\alpha_{j}^{m}}\right)_{g}^{\prime}(t) .
$$

But

$$
\begin{aligned}
\left(u_{i_{\varepsilon}+\alpha_{j}^{m}}\right)_{g}^{\prime}(t) & \in F\left(t, u_{i_{\varepsilon}+\alpha_{j}^{m}}(t)\right)-p(t) u_{i_{\varepsilon}+\alpha_{j}^{m}}(t) \\
& \subset F\left(t, B_{\varepsilon}(u(t))-p(t) u_{i_{\varepsilon}+\alpha_{j}^{m}}(t)\right.
\end{aligned}
$$

whence

$$
y_{m}(t) \in \operatorname{coF}\left(t, B_{\varepsilon}(u(t))-p(t) \cdot \sum_{j=1}^{\tilde{l}_{m}} \lambda_{j}^{m} u_{i_{\varepsilon}+\alpha_{j}^{m}}(t)\right.
$$

and, as $m \rightarrow \infty$,

$$
u_{g}^{\prime}(t) \in \overline{c o} F\left(t, B_{\varepsilon}(u(t))-p(t) u(t) .\right.
$$

Using Hypothesis 2.(ii) one gets

$$
u_{g}^{\prime}(t) \in F(t, u(t))-p(t) u(t)
$$

and the fact that $u$ is a solution is achieved.

To prove the compactness of the solution set, let us choose a sequence $\left(u_{n}\right)_{n}$ of solutions. For each $n \in \mathbb{N}$, one can find a selection $f_{n} \in L_{g}^{1}\left([0, T], \mathbb{R}^{d}\right)$ of $F\left(\cdot, u_{n}(\cdot)\right)$ satisfying:

$$
\left\{\begin{array}{l}
\left(u_{n}\right)_{g}^{\prime}(t)+p(t) u_{n}(t)=f_{n}(t), \quad \mu_{g} \text {-a.e. in }[0, T] \\
u_{n}(0)=u_{n}(T)
\end{array}\right.
$$

In the same way as before, $\left(f_{n}\right)_{n}$ is, by Hypothesis 3 , relatively weakly compact in $L^{1}\left([0, T], \mathbb{R}^{d}\right)$, thus one can find a subsequence (labelled with the same indexes) convergent in this topology to some $f \in L_{g}^{1}\left([0, T], \mathbb{R}^{d}\right)$ and one gets the pointwise convergence of $\left(u_{n}\right)_{n}$ towards $u:[0, T] \rightarrow \mathbb{R}^{d}$, which is the unique solution of

$$
\left\{\begin{array}{l}
u_{g}^{\prime}(t)+p(t) u(t)=f(t), \quad \mu_{g}-\text { a.e. in }[0, T], \\
u(0)=u(T) .
\end{array}\right.
$$

On the other hand, the sequences of the primitives involved when explicitly writing each $u_{n}$, namely

$$
\left(\int_{0} \frac{\alpha(s) e^{\int_{0}^{s} \tilde{p}(r) d g(r)}}{1-p(s) \mu_{g}(\{s\})} \cdot f_{n}(s) d g(s)\right)_{n}, \operatorname{resp} \cdot\left(\int^{T} \frac{\alpha(s) e^{\int_{0}^{s} \tilde{p}(r) d g(r)}}{1-p(s) \mu_{g}(\{s\})} \cdot f_{n}(s) d g(s)\right)_{n}
$$

are, due to the inequality (4) combined with Lemma 1, equi-regulated. It follows, by Theorem 1, that the convergence is uniform.

In other words, $\left(u_{n}\right)_{n}$ tends uniformly to $u:[0, T] \rightarrow \mathbb{R}^{d}$. 
Looking at the proof of the similar step of the existence part, it is clear that, similarly, it can be shown that $u$ is a solution of (1) and the compactness of the solution set is verified.

\section{Existence Results for Periodic Impulsive Inclusions without Upper Semicontinuity}

As already discussed, the theory of Stieltjes differential equations contains, in a particular case, the theory of impulsive equations. In what follows we shall deduce from our main Theorem 3 the existence of solutions for impulsive differential inclusions with periodic boundary conditions, without upper semicontinuity assumption on the multifunction on the right-hand side.

Consider thus a first order periodic differential inclusion

$$
\begin{aligned}
& u^{\prime}(t)+p(t) u(t) \in \tilde{F}(t, u(t)) \text { a.e. in }[0, T] \backslash\left\{\tau_{i}, i=1, \ldots, m\right\}, \\
& u\left(\tau_{i}+\right)-u\left(\tau_{i}\right) \in I_{i}\left(u\left(\tau_{i}\right)\right), i=1, \ldots, m \\
& u(0)=u(T),
\end{aligned}
$$

with multivalued jumps $I_{i}: \mathbb{R}^{n} \rightarrow \mathcal{P}\left(\mathbb{R}^{n}\right), i=1, \ldots, m$, where $0<\tau_{1}<\cdots<\tau_{m}<T$ and $\tilde{F}:[0, T] \times \mathbb{R}^{n} \rightarrow \mathcal{P}\left(\mathbb{R}^{n}\right)$.

This problem can be seen as a measure differential problem of type (1) with

$$
\begin{aligned}
g(t) & =t+\sum_{i=1}^{m} \chi_{\left(\tau_{i}, T\right]}(t), \\
F(t, u) & = \begin{cases}\tilde{F}(t, u), & \text { if } t \in[0, T] \backslash\left\{\tau_{i}, i=1, \ldots, m\right\}, \\
I_{i}(u), & \text { if } t=\tau_{i}, i=1, \ldots, m\end{cases}
\end{aligned}
$$

as a consequence of the definition of the $g$-derivative:

$$
u_{g}^{\prime}\left(\tau_{i}\right)=\frac{u\left(\tau_{i}+\right)-u\left(\tau_{i}\right)}{g\left(\tau_{i}+\right)-g\left(\tau_{i}\right)}=u\left(\tau_{i}+\right)-u\left(\tau_{i}\right) \text { for } i=1, \ldots, m .
$$

Traditionally, a function $u:[0, T] \rightarrow \mathbb{R}^{d}$ is a solution of the specified impulsive problem if it is continuous at every $t \in[0, T] \backslash\left\{\tau_{1}, \ldots, \tau_{m}\right\}$, left continuous at every $t \in\left\{\tau_{1}, \ldots, \tau_{m}\right\}$, it has right limit at every point $\tau_{i}, i=1, \ldots, m$, and, of course, it satisfies the conditions in (5).

We derive from Theorem 3 the following existence result.

Theorem 4. Let $\tilde{F}:[0, T] \times \mathbb{R}^{d} \rightarrow \mathcal{P}\left(\mathbb{R}^{d}\right)$ satisfy the following hypotheses.

Hypothesis 4. For every regulated function $u:[0, T] \rightarrow \mathbb{R}^{d}, \tilde{F}(\cdot, u(\cdot))$ has measurable selections.

Hypothesis 5. (i) for every $i=1, \ldots, m, I_{i}: \mathbb{R}^{d} \rightarrow \mathcal{P}\left(\mathbb{R}^{d}\right)$ is convex, compact valued and it is upper semicontinuous; besides, there exist some constants $M_{1}, \ldots, M_{m}$ such that for each $i \in\{1, \ldots, m\}$

$$
\left|I_{i}(u)\right| \leq M_{i}, \text { for all } u \in \mathbb{R}^{d} ;
$$

(ii) for a.e. $t \in[0, T] \backslash\left\{\tau_{1}, \ldots, \tau_{m}\right\}, \tilde{F}(t, \cdot)$ is convex, compact-valued and upper semicontinuous on $\mathbb{R}^{d} \backslash K(t)$, the set $K(t)$ being empty or the union

$$
K(t)=\bigcup_{j: t \in \mathcal{I}_{j}} K_{j}(t)
$$

where each $K_{j}$ satisfies condition (3) with $D K_{j}(t, u)(1)$ instead of $D_{g} K_{j}(t, u)$. 
Hypothesis 6. There exists a function $\tilde{\phi}$, Lebesgue-integrable on $[0, T]$, such that

$$
|\tilde{F}(t, u)| \leq \tilde{\phi}(t)
$$

for almost all $t \in[0, T] \backslash\left\{\tau_{1}, \ldots, \tau_{m}\right\}$ and all $u \in \mathbb{R}^{d}$.

Then the periodic differential inclusion (5) admits solutions.

Proof. Since all the other hypotheses in Theorem 3 are easy to check, let us only note that $\phi:[0, T] \rightarrow \mathbb{R}$, defined as

$$
\phi(t)=\left\{\begin{array}{l}
\tilde{\phi}(t), \text { if } t \in[0, T] \backslash\left\{\tau_{i}, i=1, \ldots, m\right\} \\
M_{i}, \text { if } t=\tau_{i}, i=1, \ldots, m
\end{array}\right.
$$

satisfies Hypothesis 6.

But, what is more, from our main result we can deduce the existence of solutions for the first order differential inclusions with periodic boundary conditions when infinitely many instantaneous modifications occur in the behavior of the process, more precisely when there are countably many impulses. We thus significantly generalize the known results, since for periodic impulsive boundary value problems this case is not, as far as the authors know, covered in the literature.

We are thus concerned with

$$
\begin{aligned}
& u^{\prime}(t)+p(t) u(t) \in \tilde{F}(t, u(t)) \text { a.e. in }[0, T] \backslash\left\{\tau_{i}, i \in \mathbb{N}\right\} \\
& u\left(\tau_{i}+\right)-u\left(\tau_{i}\right) \in I_{i}\left(u\left(\tau_{i}\right)\right), i \in \mathbb{N} \\
& u(0)=u(T)
\end{aligned}
$$

with multivalued jumps $I_{i}: \mathbb{R}^{n} \rightarrow \mathcal{P}\left(\mathbb{R}^{n}\right), i \in \mathbb{N}$, where $0<\tau_{1}<\cdots<\tau_{m}<\ldots<T$ and $\tilde{F}:[0, T] \times \mathbb{R}^{n} \rightarrow \mathcal{P}\left(\mathbb{R}^{n}\right)$. Suppose that the set of points where $\left(\tau_{i}\right)_{i}$ accumulate is of null Lebesgue measure.

We can write it as a measure differential problem of type (1) with

$$
\begin{aligned}
g(t) & =t+\sum_{i=1}^{\infty} \frac{1}{2^{i}} \chi_{\left(\tau_{i}, T\right]}(t), \\
F(t, u) & = \begin{cases}\tilde{F}(t, u), & \text { if } t \in[0, T] \backslash\left\{\tau_{i}, i \in \mathbb{N}\right\}, \\
2^{i} I_{i}(u), & \text { if } t=\tau_{i}, i \in \mathbb{N} .\end{cases}
\end{aligned}
$$

In this framework, we can deduce the following result.

Theorem 5. Let $\tilde{F}:[0, T] \times \mathbb{R}^{d} \rightarrow \mathcal{P}\left(\mathbb{R}^{d}\right)$ satisfy the following hypotheses.

Hypothesis 7. For every regulated function $u:[0, T] \rightarrow \mathbb{R}^{d}, \tilde{F}(\cdot, u(\cdot))$ has measurable selections.

Hypothesis 8. (i) for every $i \in \mathbb{N}, I_{i}: \mathbb{R}^{d} \rightarrow \mathcal{P}\left(\mathbb{R}^{d}\right)$ is convex, compact valued and it is upper semicontinuous; besides, there exist some constants $M_{i}, i \in \mathbb{N}$ with $\sum_{i=1}^{\infty} M_{i}<\infty$ such that for each $i \in\{1, \ldots, m\}$

$$
\left|I_{i}(u)\right| \leq M_{i}, \text { for all } u \in \mathbb{R}^{d} ;
$$

(ii) for a.e. $t \in[0, T] \backslash\left\{\tau_{i}, i \in \mathbb{N}\right\}, \tilde{F}(t, u)$ is convex and compact for all $u \in \mathbb{R}^{d} \backslash K(t)$ and $\tilde{F}(t, \cdot)$ is upper semicontinuous on $\mathbb{R}^{d} \backslash K(t)$, the set $K(t)$ being empty or the union

$$
K(t)=\bigcup_{j: t \in \mathcal{I}_{j}} K_{j}(t)
$$


where $K_{j}, j \in \mathbb{N}$ satisfy the condition (3) with $D K_{j}(t, u)(1)$ replacing $D_{g} K_{j}(t, u)$.

Hypothesis 9. There exists a function $\tilde{\phi}$, Lebesgue-integrable on $[0, T]$, such that

$$
|\tilde{F}(t, u)| \leq \tilde{\phi}(t)
$$

for almost all $t \in[0, T] \backslash\left\{\tau_{i}, i \in \mathbb{N}\right\}$ and all $u \in \mathbb{R}^{d}$.

Then the periodic differential inclusion (6) admits solutions.

Proof. It is enough to note that, when defining the set $E$ in the proof of Theorem 3, we can suppose that it includes the set of accumulation points of $\left(\tau_{i}\right)_{i}$ which are not impulsive points (this is $\mu_{g}$-null), while, at the accumulation points belonging to $\left\{\tau_{i}: i \in \mathbb{N}\right\}$, the upper semicontinuity and the convexity and compactness of the values are checked. By the expression on $g$ in this case, obviously at any $t$ which is not a point where $\tau_{i}$ accumulate, $D_{g} K_{j}(t, u)=D K_{j}(t, u)(1)$.

Let us study the following periodic impulsive problem without upper semicontinuity.

Example 1. Consider the problem

$$
\begin{aligned}
& u^{\prime}(t)+p(t) u(t) \in \tilde{F}(t, u(t)) \text { a.e. in }[0,1] \backslash\left\{\tau_{i}, i \in \mathbb{N}\right\}, \\
& u\left(\tau_{i}+\right)-u\left(\tau_{i}\right) \in I_{i}\left(u\left(\tau_{i}\right)\right), i \in \mathbb{N} \\
& u(0)=u(1),
\end{aligned}
$$

where

$$
\tau_{i}=\frac{1}{2}+\frac{1}{2+i}, \text { for every } i \in \mathbb{N}
$$

and let $\tilde{F}:[0,1] \times \mathbb{R} \rightarrow \mathbb{R}$ be the multifunction given in [4] (Example 3.11), which is not upper semicontinuous, nor convex compact-valued on a dense subset of $\mathbb{R}$, but satisfies the Hypotheses 7 , 8(ii) and 9 in Theorem 5. If each $I_{i}: \mathbb{R} \rightarrow \mathcal{P}(\mathbb{R})$ is defined by

$$
I_{i}(u)=\overline{B_{\frac{1}{2^{i}}}(0)}, \text { for all } u \in \mathbb{R},
$$

it is easy to see that it satisfies the Hypothesis 8(i) so the existence of solutions for the considered problem is guaranteed by Theorem 5 . Let us notice that there are countably many impulse moments that accumulate at the middle of the interval, so, it is a hybrid system with a Zeno behavior, which cannot be studied using the classical theory of impulsive differential inclusions.

Remark 1. It is easy to construct from here an example of Stieltjes differential problem of type (1) for which Theorem 3 applies, by considering $g$ and $F$ given by (7).

\section{Existence Result for Periodic Dynamic Inclusions on Time Scales without Upper Semicontinuity}

We prove in this section that our Theorem 3 also yields an existence result for dynamic inclusions on time scales with periodic boundary conditions (for a comprehensive introduction in the time scales theory, see [36]).

Let $\mathbb{T} \subset[0, T]$ be a bounded time scale (i.e., a nonempty and closed subset of $\mathbb{R}$, with the standard topology inherited from $\mathbb{R}$ ). Suppose $0=\min \mathbb{T}, T=\max \mathbb{T}$. For two points $a, b$ in $\mathbb{T}$, let $[a, b)_{\mathbb{T}}=\{t \in \mathbb{T}: a \leq t<b\}$ be the time scales interval.

The forward jump operator $\sigma: \mathbb{T} \rightarrow \mathbb{T}$ is defined by $\sigma(t)=\inf \{s \in \mathbb{T}: s>t\}$. By convention, $\sigma(T)=T$.

A point $t \in \mathbb{T}$ is called right-dense, resp. right-scattered, if $\sigma(t)=t$, resp. $\sigma(t)>t$. It is known (Lemma 3.1 in [37]) that the set of right-scattered points of $\mathbb{T}$ is at most countable; let $\tau_{i}, i \in \mathbb{N}$ be its elements. Suppose that the set of right-dense points where the right-scattered 
points accumulate is of null Lebesgue measure (it happens, for instance, if $\mathbb{T}$ is a typical time scales domain, see [2]).

The Lebesgue measure on $\mathbb{T}, \mu_{\Delta}$, was introduced and studied in [37], while for the Lebesgue $\Delta$-integral we refer to [36-39] or [40].

Let us recall that, by [37] (Theorem 5.2), if $f: \mathbb{T} \rightarrow \mathbb{R}^{d}$, for any $a<b \in[0, T]$,

$$
\int_{[a, b)_{\mathbb{T}}} f(s) \Delta s=\int_{[a, b) \cap \mathbb{T}} f(s) d s+\sum_{i: \tau_{i} \in[a, b)} f\left(\tau_{i}\right)\left(\sigma\left(\tau_{i}\right)-\tau_{i}\right) .
$$

On the other hand, following [41], let $g:[0, T] \rightarrow \mathbb{R}$ be defined by

$$
g(t)=\inf \{s \in \mathbb{T}: s \geq t\}
$$

which is left-continuous and nondecreasing and satisfies

$$
g(t)=t \text { and } \sigma(t)=g(t+) \quad \text { whenever } t \in \mathbb{T} .
$$

It follows, by the definition of the Lebesgue-Stieltjes integral, that

$$
\begin{aligned}
& \int_{[a, b)]_{\mathbb{T}}} f(s) \Delta s \\
= & \int_{([a, b) \cap \mathbb{T}) \backslash\left\{\tau_{i}, i \in \mathbb{N}\right\}} f(s) d s+\int_{[a, b) \cap\left\{\tau_{i}, i \in \mathbb{N}\right\}} f(s) d s+\sum_{i: \tau_{i} \in[a, b)} f\left(\tau_{i}\right)\left(\sigma\left(\tau_{i}\right)-\tau_{i}\right) \\
= & \int_{([a, b) \cap \mathbb{T}) \backslash\left\{\tau_{i}, i \in \mathbb{N}\right\}} f(s) d g(s)+0+\sum_{i: \tau_{i} \in[a, b)} f\left(\tau_{i}\right)\left(g\left(\tau_{i}+\right)-g\left(\tau_{i}\right)\right) \\
= & \int_{[a, b)} f(s) d g(s) .
\end{aligned}
$$

Definition 2. The function $f: \mathbb{T} \rightarrow \mathbb{R}^{d}$ is said to be $\Delta$-differentiable at $t \in \mathbb{T}$ if there is an element $f^{\Delta}(t) \in \mathbb{R}^{d}$ (the $\Delta$-derivative of $f$ at $t$ ) such that for any $\varepsilon>0$ there is a neighborhood of $t$ such that

$$
\left\|f(\sigma(t))-f(s)-f^{\Delta}(t)[\sigma(t)-s]\right\| \leq \varepsilon|\sigma(t)-s| \text { on this neighborhood. }
$$

In [39] (Theorem 1.3) it was proved that:

(i) $f$ is continuous at any point where it is $\Delta$-differentiable;

(ii) at a right-scattered point where $f$ is left-continuous, it is $\Delta$-differentiable and

$$
f^{\Delta}(t)=\frac{f(\sigma(t))-f(t)}{\sigma(t)-t} .
$$

In this section, the focus is on a dynamic inclusion on time scales with periodic boundary condition

$$
\left\{\begin{array}{l}
u^{\Delta}(t)+p(t) u(t) \in \bar{F}(t, u(t)), t \in \mathbb{T} \\
u(0)=u(T)
\end{array}\right.
$$

where $p: \mathbb{T} \rightarrow \mathbb{R}$ is Lebesgue $\Delta$-integrable and $\bar{F}: \mathbb{T} \times \mathbb{R}^{d} \rightarrow \mathcal{P}\left(\mathbb{R}^{d}\right)$.

We say that $u: \mathbb{T} \rightarrow \mathbb{R}^{d}$ is a solution for the considered problem if it is $\Delta$-absolutely continuous, there exists a selection $f$ of $\bar{F}(\cdot, u(\cdot))$ Lebesgue $\Delta$-integrable with $u^{\Delta}(t)+$ $p(t) u(t)=f(t), \mu_{\Delta}$-a.e. and $u(0)=u(T)$. For completeness, let us recall [10] that $u$ is $\Delta$-absolutely continuous if for every $\varepsilon>0$ there is $\delta_{\varepsilon}>0$ such that

$$
\sum_{j=1}^{m}\left\|u\left(t^{\prime \prime}{ }_{j}\right)-u\left(t_{j}^{\prime}\right)\right\|<\varepsilon
$$

for any set $\left\{\left(t_{j}^{\prime}, t^{\prime \prime}{ }_{j}\right)\right\}_{i=1}^{m}$ of non-overlapping intervals with $t_{j}^{\prime}, t^{\prime \prime}{ }_{j} \in \mathbb{T}$ and $\sum_{j=1}^{m}\left(t^{\prime \prime}{ }_{j}-t_{j}^{\prime}\right)<\delta_{\varepsilon}$. 
For any function $u: \mathbb{T} \rightarrow \mathbb{R}^{d}$ we define its Slavik extension [41]

$$
u^{g}:[0, T] \rightarrow \mathbb{R}^{d}, \quad u^{g}(t)=u(g(t)) .
$$

Let us also extend, in the same way, the multifunction $\bar{F}$ in order to get $\bar{F}^{g}:[0, T] \times \mathbb{R}^{d} \rightarrow$ $\mathcal{P}\left(\mathbb{R}^{d}\right), \bar{F}^{g}(t, u)=\bar{F}(g(t), u)$.

Lemma 3. A function $u: \mathbb{T} \rightarrow \mathbb{R}^{d}$ is a solution of problem (9) if and only if $u^{g}$ is a solution of

$$
\left\{\begin{array}{l}
u_{g}^{\prime}(t)+p(t) u(t) \in \bar{F}^{g}(t, u(t)), \mu_{g} \text {-a.e. } t \in[0, T] \\
u(0)=u(T)
\end{array}\right.
$$

which is a problem of type (1).

Proof. Let $u$ be a solution of (9). Then one can find a selection $f$ of $\bar{F}(\cdot, u(\cdot))$, Lebesgue $\Delta$-integrable on $\mathbb{T}$, such that

$$
u^{\Delta}(t)+p(t) u(t)=f(t), t \in \mathbb{T} .
$$

Since if $u$ is left-continuous at the right-scattered points one can see (as in [2]) that, for every $t \in \mathbb{T}$,

$$
u^{\Delta}(t)=\left(u^{g}\right)_{g}^{\prime}(t),
$$

so it follows that $\left(u^{g}\right)_{g}^{\prime}(t)+p(t) u^{g}(t)=f(t)$ for every $t \in \mathbb{T}$. At the same time, the $g$-derivative of $u^{g}$ does not make sense on $[0, T] \backslash \mathbb{T}$ as $g$ is constant, but $\mu_{g}([0, T] \backslash \mathbb{T})=0$, therefore

$$
\left(u^{g}\right)_{g}^{\prime}(t)+p(t) u^{g}(t)=f(t), \quad \mu_{g}-\text { a.e. } t \in[0, T]
$$

and on the set where the equality holds,

$$
f(t) \in \bar{F}(t, u(t))=\bar{F}\left(g(t), u^{g}(t)\right)=\bar{F}^{g}\left(t, u^{g}(t)\right)
$$

since $g(t)=t$ on $\mathbb{T}$.

Also, $u^{g}(0)=u(0)$ and $u^{g}(T)=u(T)$, so $u^{g}$ is indeed a solution of (10).

Conversely, let $y$ be a solution of (10). Then the function $u: \mathbb{T} \rightarrow \mathbb{R}^{d}$ given by $u(t)=y(t)$ for every $t \in \mathbb{T}$ is a solution on the dynamic problem (9) since

$$
u^{\Delta}(t)=\left(y^{g}\right)_{g}^{\prime}(t)=y_{g}^{\prime}(t), \text { for all } t \in \mathbb{T} .
$$

This characterization allows us to deduce the existence of solutions of (9) from Theorem 3.

Theorem 6. Let $\bar{F}: \mathbb{T} \times \mathbb{R}^{d} \rightarrow \mathcal{P}\left(\mathbb{R}^{d}\right)$ satisfy the assumptions below.

Hypothesis 10. For every regulated function $u: \mathbb{T} \rightarrow \mathbb{R}^{d}, \bar{F}(\cdot, u(\cdot))$ has measurable selections.

Hypothesis 11. (i) for every right-scattered $t \in \mathbb{T}, \bar{F}(t, \cdot)$ is convex, compact valued and it is upper semicontinuous;

(ii) for $\mu_{\Delta}$-a.e. right-dense $t \in \mathbb{T}, \bar{F}(t, \cdot)$ is convex compact-valued and upper semicontinuous on $\mathbb{R}^{d} \backslash K(t)$, the set $K(t)$ being empty or the union

$$
K(t)=\bigcup_{j: t \in \mathcal{I}_{j}} K_{j}(t),
$$


where for each $j \in \mathbb{N}, K_{j}: \mathbb{T} \rightarrow \mathcal{P}\left(\mathbb{R}^{d}\right)$ satisfies the inclusion (3) with $D K_{j}(t, u)(1)$ instead of $D_{g} K_{j}(t, u)$;

Hypothesis 12. there exists a function $\phi$, Lebesgue $\Delta$-integrable on $\mathbb{T}$, such that

$$
|\bar{F}(t, u)| \leq \phi(t)
$$

for $\mu_{\Delta}$-almost all $t \in \mathbb{T}$ and all $u \in \mathbb{R}^{d}$.

Then the dynamic inclusion with periodic boundary conditions (9) has solutions.

Proof. When $\bar{F}$ satisfies our assumptions, $\bar{F}^{g}$ satisfies the hypotheses of Theorem 3 . To see this, it suffices to note that, when defining the set $E$ in the proof of Theorem 3, we may consider that it contains the right-dense points where $\left(\tau_{i}\right)_{i}$ accumulate (this is a $\mu_{\Delta}$-null set). At the accumulation points belonging to $\left\{\tau_{i}: i \in \mathbb{N}\right\}$ we have upper semicontinuity and convex compact values. Finally, at any $t$ which is not a point where $\tau_{i}$ accumulate, $D_{g} K_{j}(t, u)=D K_{j}(t, u)(1)$.

It follows that the Stieltjes differential problem (10) has at least one solution whence, by Lemma 3, the dynamic problem on time scales (9) has solutions.

Following the same idea as in Example 1, let us see a periodic dynamic problem on time scales without upper semicontinuity for which the existence of solutions can be provided.

Example 2. Consider the time scale domain

$$
\mathbb{T}=\left[0, \frac{1}{2}\right] \cup\left\{\frac{1}{2}+\frac{1}{2+i} ; i \in \mathbb{N}\right\}
$$

and the impulsive problem

$$
\left\{\begin{array}{l}
u^{\Delta}(t)+p(t) u(t) \in \bar{F}(t, u(t)), t \in \mathbb{T} \\
u(0)=u(1)
\end{array}\right.
$$

where $p \in L_{\Delta}^{1}(\mathbb{T})$ and $\bar{F}: \mathbb{T} \times \mathbb{R} \rightarrow \mathbb{R}$ is the multifunction given in [4] (Example 3.11) for $t \in\left[0, \frac{1}{2}\right]$, respectively

$$
\bar{F}(t, u)=\overline{B_{\frac{1}{2^{i}}}(0)}, \quad \text { if } t=\frac{1}{2}+\frac{1}{2+i^{\prime}}, i \in \mathbb{N} .
$$

The set of right-dense points where the right-scattered points $\frac{1}{2}+\frac{1}{2+i}, i \in \mathbb{N}$ accumulate is of null Lebesgue measure (it consists in $\left\{\frac{1}{2}\right\}$ ).

F obviously satisfies the Hypotheses 10 and 12 in Theorem 6.

Besides, for any right-scattered point (i.e., for any $\frac{1}{2}+\frac{1}{2+i}, i \in \mathbb{N}$ ), $\bar{F}(t, \cdot)$ is compact convexvalued and constant, thus upper semicontinuous.

At almost any right-dense point (i.e., almost everywhere in $\left.\left[0, \frac{1}{2}\right]\right), \bar{F}(t, \cdot)$ is not upper semicontinuous, nor convex compact-valued on a dense subset of $\mathbb{R}$, but satisfies the Hypothesis 11(ii), thus the existence of solutions for the considered problem is a consequence of Theorem 6.

\section{Conclusions}

In this work, an existence theory was developed for first-order differential inclusions with periodic boundary conditions, leading to a very general result (Theorem 3 ) due to the substitution of the usual derivative of the state with the Stieltjes derivative related to a nondecreasing function. For a particular non-decreasing function, the existence of solutions of impulsive differential problems with finitely or countably many impulsive 
moments and set-valued impulses was derived (Theorem 4, respectively 5). Furthermore, being aware of the tight connection between the theory of Stieltjes differential equations and that of dynamic equations on time scales, an existing result for dynamic inclusions on time scales with periodic conditions on the boundary was provided (Theorem 6). Future directions connected to this work could focus on generalized differential equations with similar conditions on the boundary.

Besides the generality of our outcome following from the remark that the measure differential equations encompass a large number of classical problems (such as ordinary differential equations, difference equations, impulsive and generalized differential equations), we highlight that this is the first study of differential inclusions with periodic boundary conditions involving Stieltjes derivative where the hypotheses on the right-hand side to have convex compact values and to be upper semicontinuous are relaxed.

Author Contributions: The authors contributed equally to this work. All authors have read and agreed to the published version of the manuscript.

Funding: This research received no external funding.

Institutional Review Board Statement: Not applicable.

Informed Consent Statement: Not applicable.

Data Availability Statement: Not applicable.

Acknowledgments: The authors are grateful to the reviewers for their constructive remarks.

Conflicts of Interest: The authors declare no conflict of interest.

\section{References}

1. Pouso, R.L.; Rodriguez, A. A new unification of continuous, discrete, and impulsive calculus through Stieltjes derivatives. Real Anal. Exch. 2015, 40, 319-353. [CrossRef]

2. Frigon, M.; Pouso, R.L. Theory and applications of first-order systems of Stieltjes differential equations. Adv. Nonlinear Anal. 2017, 6, 13-36. [CrossRef]

3. Monteiro, G.A.; Satco, B. Extremal solutions for measure differential inclusions via Stieltjes derivatives. Adv. Differ. Equ. 2019, 239, 1-18. [CrossRef]

4. $\quad$ Pouso, R.L.; Marquez Albes, I.M.; Rodriguez-Lopez, J. Solvability of non-semicontinuous systems of Stieltjes differential inclusions and equations. Adv. Differ. Equ. 2020, 2020, 227. [CrossRef]

5. Cao, Y.; Sun, J. On existence of nonlinear measure driven equations involving non-absolutely convergent integrals. Nonlinear Anal. Hybrid Syst. 2016, 20, 72-81. [CrossRef]

6. Di Piazza, L.; Marraffa, V.; Satco, B. Approximating the solutions of differential inclusions driven by measures. Ann. Mat. Pura Appl. 2019, 198, 2123-2140. [CrossRef]

7. Federson, M.; Mesquita, J.G.; Slavik, A. Measure functional differential equations and functional dynamic equations on time scales. J. Differ. Equ. 2012, 252, 3816-3847. [CrossRef]

8. Monteiro, G.A.; Satco, B. Distributional, differential and integral problems: Equivalence and existence results. Electron. J. Qual. Theory Differ. Equ. 2017, 7, 1-26. [CrossRef]

9. Cichoń, M.; Satco, B.; Sikorska-Nowak, A. Impulsive nonlocal differential equations through differential equations on time scales. Appl. Math. Comp. 2011, 218, 2449-2458. [CrossRef]

10. Frigon, M.; Gilbert, H. Systems of first order inclusions on time scales. Topol. Methods Nonlinear Anal. 2011, 37, 147-163.

11. Aubin, J.P.; Cellina, A. Differential Inclusions. In Set-Valued Maps and Viability Theory; Springer: Berlin/Heidelberg, Germany, 1984.

12. Castaing, C.; Valadier, M. Convex Analysis and Measurable Multifunctions; Springer: Berlin/Heidelberg, Germany, $1977 ;$ Volume 580.

13. Djebali, S.; Gorniewicz, L.; Ouahab, A. Existence and Structure of solution sets for impulsive differential inclusions: A survey. Lect. Notes Nonlinear Anal. 2012, 13.

14. Graef, J.R.; Henderson, J.; Ouahab, A. Impulsive Differential Inclusions. A Fixed Point Approach; de Gruyter: Berlin, Germany, 2013.

15. Satco, B. Existence results for Urysohn integral inclusions involving the Henstock integral. J. Math. Anal. Appl. 2007, 336, 44-53. [CrossRef]

16. Satco, B.; Smyrlis, G. Periodic boundary value problems involving Stieltjes derivatives. J. Fixed Point Theory Appl. 2020, $22,24$. [CrossRef]

17. Satco, B.; Smyrlis, G. Applications of Stieltjes Derivatives to Periodic Boundary Value Inclusions. Mathematics 2020, 8, 2142. [CrossRef] 
18. Lygeros, J.; Tomlin, C.; Sastry, S. Hybrid Systems: Modeling, Analysis and Control; Electronic Research Laboratory, University of California: Berkeley, CA, USA, 2008.

19. Bainov, D.; Simeonov, P. Impulsive Differential Equations: Periodic Solutions and Applications; Routledge: New York, NY, USA, 1993.

20. Dhage, B.C.; Boucherif, A.; Ntouyas, N.K. On periodic boundary value problems of first-order perturbed impulsive differential inclusions. Electron. J. Differ. Equ. 2004, 84, 1-9.

21. Graef, J.R.; Ouahab, A. First order impulsive differential inclusions with periodic conditions. Theory Differ. Equ. 2008, 31, 1-40. [CrossRef]

22. Frigon, M.; O'Regan, D. Impulsive differential equations with variable times. Nonlin. Anal. TMA 1996, 26, 1913-1922. [CrossRef]

23. Henderson, J.; Ouahab, A. Impulsive differential inclusions with fractional order. Comput. Math. Appl. 2010, 59, 1191-1226. [CrossRef]

24. Cardinali, P.; Servadei, R. Periodic solutions of nonlinear impulsive differential inclusions with constraints. Proc. Am. Math. Soc. 2004, 132, 2339-2349. [CrossRef]

25. Kryszewski, W.; Plaskacz, S. Periodic solutions to impulsive differential inclusions with constraints. Nonlinear Anal. 2006, 65, 1794-1804. [CrossRef]

26. Atici, F.M.; Biles, D.C. First order dynamic inclusions on time scales. J. Math. Anal. Appl. 2004, 292, 222-237. [CrossRef]

27. Satco, B.; Turcu, C. First order multivalued problems on time scales. Fixed Point Theory 2013, 14, 483-496.

28. Dai, Q.; Tisdell, C.C. Existence of Solutions to first-order dynamic boundary value problems. Int. J. Differ. Equ. 2006, 1, 1-17.

29. Sun, J.P.; Li, W.T. Existence of solutions to nonlinear first-order PBVPs on time scales. Nonlin. Anal. 2007, 67, 883-888. [CrossRef]

30. Fraňková, D. Regulated functions. Math. Bohem. 1991, 116, 20-59. [CrossRef]

31. Young, W.H. On integrals and derivatives with respect to a function. Proc. London Math. Soc. 1917, 1, 35-63. [CrossRef]

32. Frigon, M.; Tojo, F.A.F. Stieltjes differential systems with nonmonotonic derivators. Boundary Value Problems 2020, 1, 1-24. [CrossRef]

33. Pouso, R.L.; Márquez, I.A. General existence principles for Stieltjes differential equations with applications to mathematical biology. J. Differ. Equ. 2018, 264, 5388-5407. [CrossRef]

34. Ward, A.J. The Perron-Stieltjes integral. Math. Z. 1936 41, 578-604. [CrossRef]

35. Luc, D.T. Contingent derivatives of set-valued maps and applications to vector optimization. Math. Program. 1991, 50,99-111. [CrossRef]

36. Bohner, M.; Peterson, A. Dynamic Equations on Time Scales, An Introduction with Applications; Springer Science \& Business Media: Boston, MA, USA, 2001.

37. Cabada, A.; Vivero, D.R. Expression of the Lebesgue $\Delta$-integral on time scales as a usual Lebesgue integral; application to the calculus of $\Delta$-antiderivatives. Math. Comp. Modell. 2006, 43, 194-207. [CrossRef]

38. Bohner, M.; Guseinov, G.S. Riemann and Lebesgue integration. In Advances in Dynamic Equations on Time Scales; Bohner, M., Peterson, A.C., Eds.; Birkhäuser Boston: Boston, MA, USA, 2003; pp. 117-163.

39. Bohner, M.; Peterson, A. Advances in Dynamic Equations on Time Scales; Birkhäuser: Boston, MA, USA, 2003.

40. Guseinov, G.S. Integration on time scales. J. Math. Anal. Appl. 2003, 285, 107-127. [CrossRef]

41. Slavik, A. Dynamic equations on time scales and generalized ordinary differential equations. J. Math. Anal. Appl. 2012, 385, 534-550. [CrossRef] 